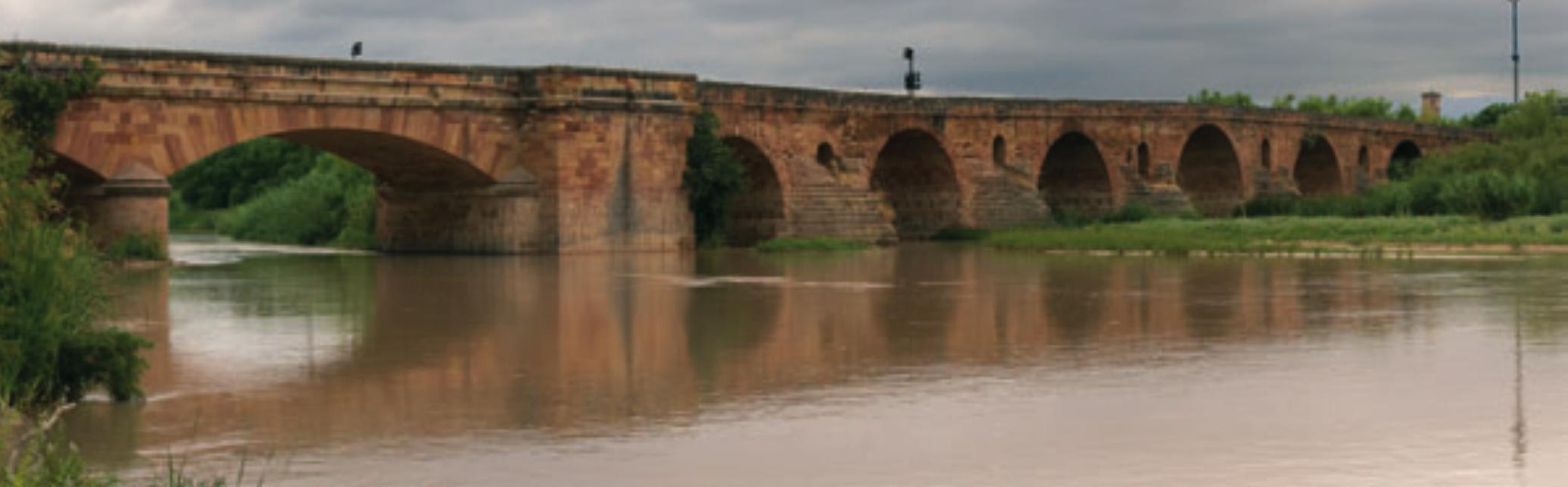

Puente romano de Andújar (Jaén). Foto: Juan Carlos Cazalla, IAPH 


\section{Infraestructuras del transporte: los puentes}

Francisco Javier Rubiato Lacambra, Dpto. Ingeniería Civil: Ordenación del Territorio, Urbanismo y Medio Ambiente, Universidad Politécnica de Madrid

\section{EL Río}

Antes de centrarnos en las infraestructuras de transporte en el Guadalquivir hay que hacer unas breves reflexiones sobre el propio río. Como todos sabemos el Guadalquivir es el eje geográfico de Andalucía. A modo de columna vertebral, estructura el territorio de Este a Oeste, a pesar de dar sus primeros pasos en dirección Nordeste, trayectoria que cambia al recibir al Hornos. De ser un pequeño río en la Sierra de Cazorla, fluyendo entre montañas, pasa poco después, en su singular discurrir, a constituirse en límite entre Sierra Morena y la Campiña, para una vez pasada la ciudad de Córdoba, centrar su posición en el valle, y llegar así hasta la desembocadura. Esta disposición dentro del territorio de nuestra región llevó desde antiguo a que el río se constituyera, primero como vía de penetración, y después en eje de comunicación en el territorio. Con más importancia, si se tienen en cuenta las dificultades topográficas que representan las cordilleras Béticas y la complicada disposición de las sierras que las conforman.

Por lo expuesto no es de extrañar que desde tiempos remotos los márgenes del río fueran utilizados como vías de comunicación. Uso éste que quedo definitivamente fijado con la dominación romana, primero con la vía Hercúlea y luego con la vía Augusta, que comunicaban directamente nuestras tierras con la capital del Imperio, como aun queda atestiguado en los miliarios. Pero no solo los márgenes fueron utilizados para la comunicación. El propio cauce sirvió durante siglos como vía. La escasa pendiente facilitaba su uso. Existen referencias a que en tiempos de los musulmanes se podía llegar navegando hasta Villa del Río, límite oriental de la actual provincia de Córdoba, y que allí una cadena tendida sobre el cauce señalaba el final de dicha posibilidad. Los constantes acarreos de materiales propiciados por la misma corriente, la diferente pluviosidad actual y el desarrollo de las técnicas del transporte, han conllevado al abandono de esa práctica. Hoy solo en uso desde la desembocadura hasta Sevilla. Aunque no hace tanto, en la segunda década del siglo pasado, en concreto en 1919 el ingeniero Carlos Mendoza presento al ministro de Fomento su Proyecto de Canalización y aprovechamiento de energía del Guadalquivir entre Córdoba y Sevilla, en el que aprovechaba los
$88 \mathrm{~m}$ de desnivel existente en los $177 \mathrm{~km}$ de longitud del tramo con once presas y sus correspondientes esclusas para permitir la navegación (AGUILÓ, 2002: 113).

\section{CAMINOS Y PUENTES}

Desde tiempos remotos la fertilidad de las tierras aportadas por el río ha favorecido la ocupación del territorio. A su vez el poblamiento y la topografía del valle han propiciado las comunicaciones y con ellas la creación de un sin fin de caminos. Es en este momento cuando el río, además de eje integrador, separa. La necesidad de continuar el camino hace imprescindible la presencia del puente.

El Guadalquivir es un río con un gran patrimonio en puentes. En el aparecen muy bien representadas todas las etapas histórico constructivas. Desde los remotos tiempos de los romanos hasta la actualidad. No se puede olvidar que la Bética fue una de las primeras regiones que se civilizó y urbanizó, y que por aqui han pasado gran número de pueblos. Con los puentes, el río intensifica su capacidad de integración del territorio: une ambas orillas, pone en contacto a las gentes, reúne tierras y crea un paisaje común.

Los puentes, desde siempre, y estén donde estén, nuestro río no es una excepción, son el resultado del esfuerzo común de muchos ciudadanos anónimos, que desde la simple aportación económica al esfuerzo físico e intelectual han contribuido a fomentar las comunicaciones, los intercambios, el comercio. Estas estructuras (los puentes) son el resultado de la superación constante. De ahí que en ellos aparezcan reflejados los más importantes avances técnicos y científicos de los pueblos que los construyeron, así como son fruto también de su pensamiento estético y reflejan fielmente la situación económica, política y social de cada momento, es decir su historia.

\section{PUENTES ANTIGUOS}

Como antes se ha dicho, en el Guadalquivir los puentes se han construido desde tiempos remotos. No queda ningún resto ante- 
rior a Roma. Es probable que existiera alguna obra, quizá próxima al nacimiento. De este modo los puentes más antiguos que han llegado hasta nosotros corresponden a este periodo. Con su política de expansión territorial, Roma creó una vasta red de vías y caminos. Como consecuencia se facilitaron las comunicaciones como nunca se había conseguido. Además, se produjo, por primera vez, más allá de la urbanización de nuevos núcleos de población, una ordenación del territorio peninsular y, en el caso que nos ocupa, de la Bética (SÁENZ, 1984: 138-153).

Dentro de la estructura viaria romana los puentes se constituyen como elementos singulares. Su aparejo es siempre de fábrica de piedra, cortada en grandes sillares, utilizando en el interior de las pilas y en las fundaciones el hormigón. Estas siempre solian coincidir con el terreno más consistente de la zona ya que colocaban los apoyos preferiblemente sobre roca (VITRUVIO POLIÓN, 1995: Libro III, Cap. IV). Utilizaban las bóvedas de cañón de modo sistemático. Éstas se apoyaban sobre robustas pilas, en ocasiones con una anchura exagerada en relación al vano, muchas veces se superaba el tercio de éste, lo que podía resultar problemático pues creaba una fuerte resistencia frente a la corriente. Las pilas se completaban con tajamares solo aguas arriba y sobre los mismos se situaban los sombreretes. Las bóvedas se destacaban del paramento por la disposición de las dovelas. La línea de impostas señalaba la diferencia entre el tímpano y el pretil siempre de piedra. Sobre el Guadalquivir solo quedan dos puentes de este periodo, el de Andújar y el de Córdoba. Ambos han sufrido a lo largo de la historia gran número de reparaciones e intervenciones que los han ido modificando. El segundo ha sido durante siglos el ultimo paso estable para cruzar el río antes de la desembocadura. Los tramos bajos de los ríos fueron los únicos obstáculos que los romanos no pudieron salvar. En Sevilla el paso se realizaba por medio del puente de barcas, de origen antiguo pero posiblemente posterior a la dominación romana.

No fueron los únicos que existieron sobre el río. Hay que tener presente la intensa romanización de la Bética. De este modo el actual puente de Úbeda La Vieja, puente medieval, sin duda fue en su origen una obra romana transformado muy probablemente en el siglo XIII (FERNÁNDEZ CASAD0, 1981). Lo mismo sucedió con el viejo puente de Alcolea del Río, sustituido en tiempos de Carlos III, como atestigua Ponz (1988: tomo XVI, carta sexta). También en las proximidades de Villanueva de la Reina, en Jaén, debió existir otro, como queda reflejado en los planos del proyecto del actual. A estos cinco habría que añadir la más que probable transformación del antiguo puente de las Herrerias por Ios Reyes Católicos (RUBIATO LACAMBRA, 2005:30). En cuanto a la Edad Media en nuestro territorio los musulmanes se caracterizaron por una política de aprovechamiento y restauración de los puentes romanos (PAVÓN MALDONAD0, 1990: 91). En algunas ocasiones además se añadieron elementos defensivos como la construcción de la torre de La Calahorra en el puente Romano de Córdoba (FERNÁNDEZ TROYANO, 1985: 11 y 28).

\section{RENACIMIENTO Y MODERNIDAD}

La modernidad entra en España de la mano de los Reyes Católicos. Los monarcas marcar las nuevas directrices políticas, al crearse la monarquía autoritaria frente al poder feudal. Los nuevos objetivos político-sociales llevan a los reyes a reactivar la construcción y mejora de caminos, así como la reparación y construcción de nuevos puentes se produce asi una intensa labor de modernización (NOVÍSIMA, 1980: 92), concentrándose la mayor cantidad de nuevas obras en la mitad sur del territorio, donde fue escenario privilegiado Andalucía y en concreto el Guadalquivir. Asi entre los siglos XV y XVI sobre nuestro río se construyeron cinco puentes'. Además de la corona hubo otros promotores como el obispo Alonso Suárez de la Fuente, que promovió la construcción del puente del Obispo en Baeza, e incluso en ocasiones el mismo pueblo se convirtió en promotor de la construcción del puente. El mejor ejemplo en este sentido nos lo da el de Montoro (RAMÍREZ DE LAS CASAS, 1986: 115). Los reyes conscientes de la importancia y de las dificultades del empeño eximieron del pago de tributos y gabelas a toda la localidad cordobesa.

El puente de la modernidad coincide con el renacer de la cultura y de las artes. Pero sigue siendo heredero de los hallazgos técnicos romanos. Así se estudian las obras antiguas. A pesar de ese deseo de enlace con la antigüedad, las bóvedas renacentistas se despegan de sus modelos romanos a causa de su mayor luz. Las roscas de las mismas suelen tener alguna concesión a lo decorativo, resaltando el extradós. En cuanto a la rasante de los tableros el ideal es la plana, pero no siempre se logra. En lo referente a la fábrica, el paramento es de piedra cortada en sillares lo más regulares posibles. Respecto a las pilas y sus fundaciones se buscan siempre los puntos más firmes. Estas se refuerzan con tajamares, en este periodo, situados tanto aguas arriba como abajo, triangulares los primeros y semicirculares los segundos. Una innovación de estos tiempos es la prolongación del tajamar hasta la altura de la rasante del tablero. Se crea de este modo un ensanche en el tablero denominado apartadero. Los pretiles seguirán siendo de sillería. Sobre el Guadalquivir se construyeron los puentes de Las Herrerías, Montoro, Mazuecos, el del Obispo y Marmolejo, magníficos ejemplos de ingeniería renacentista.

Es obligatorio para terminar este periodo citar las obras realizadas en el siglo XVIII. Tras un progresivo abandono de los caminos centrado en el siglo XVII, consecuencia de de causas complejas, que no es momento de citar. Se produce un nuevo esfuerzo de renovación. Fruto del cual se construyó en las inmediaciones de Córdoba el puente de Alcolea del Río. Su situación estratégica le ha hecho escenario de acontecimientos trascendentales en la historia de nuestro país. El primero durante la Guerra de la Independencia, el 17 de junio de 1808, batalla en la que el general Pedro Echeverri derroto al general Dupont. Mayor trascendencia para la evolución política de España tuvo el encuentro durante la revolución "Gloriosa" en 1868 de los ejércitos realista, capitaneado por el Marqués de Novaliches, y los sublevados dirigidos por Prim y Serrano, pues decidió el exilio de Isabel II². Se trata de un puente de gran desarrollo longitudinal. Consta de veinte bóvedas. Esta realizado en perfecta silleria. 


\section{A}

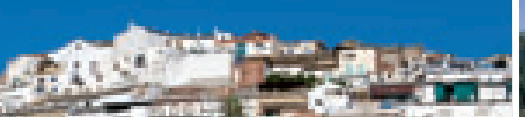
T.

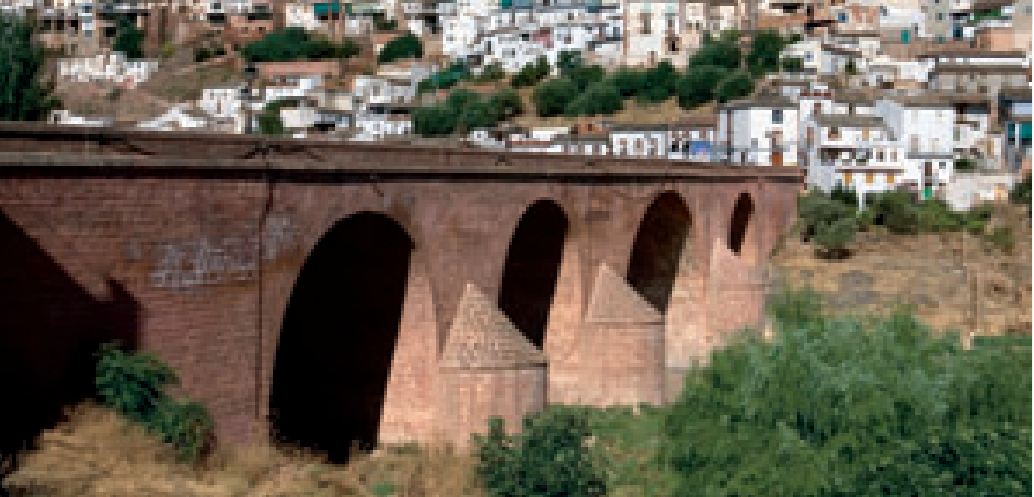
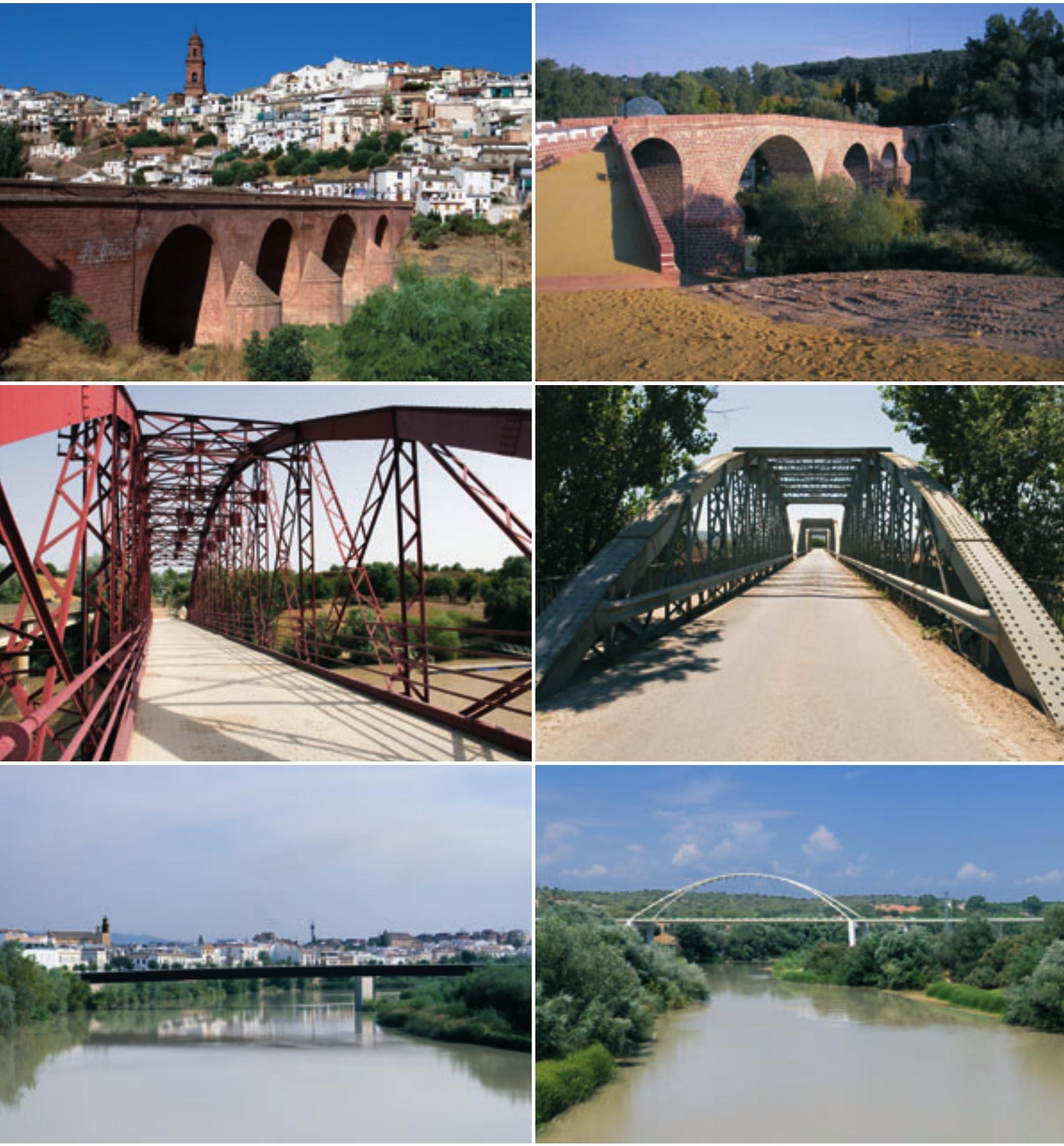

Vista de Montoro (Córdoba) con el puente romano en primer plano.

Foto: Juan Carlos Cazalla, IAPH

Puente de los Remedios. Villafranca de Córdoba. Foto: Juan Carlos Cazalla, IAPH Nuevo puente de Miraflores. Córdoba. Foto: Juan Carlos Cazalla, IAPH

Puente renacentista de Marmolejo (Jaén)

Foto: Francisco Javier Rubiato Lacambra

Puente de hierro de Villa del Río, Córdoba. Foto: Juan Carlos Cazalla, IAPH

Puente Reina Sofía en Palma del Río (Córdoba). Foto: Juan Carlos Cazalla, IAPH 


\section{EL EMPLEO DEL METAL Y LA CONSTRUCCIÓN DE PUENTES}

Aun con cierto retraso respecto de Europa, la revolución industrial marcó la centuria del siglo XIX también en nuestro país. Con ella Ilego a España el hierro que a partir de entonces se aplicará a la construcción de puentes. Con el cambiará radicalmente la concepción de los puentes. Los nuevos sistemas metálicos traerán una auténtica revolución estética. Se desarrolló toda una tipología de vigas. Como elemento decisivo en esta revolución, influyó el desarrollo de un nuevo sistema de trasporte, el ferrocarril. Aunque la nueva tipología de puentes no sea exclusiva del mismo.

En el Guadalquivir se encuentran gran número de puentes metálicos del siglo XIX, o al menos en su origen fueron de dicho siglo. El más destacado sin duda, es el puente de Isabel II en Sevilla. Construido siguiendo al puente parisino del Carrusel utiliza la cercha Polenceau (PUENTE, 1844). También es de destacar el puente de Palma del Río. Éste de vigas rectas armadas y doble celosía, formando dobles cruces de San Andrés (PROYECTO: caja 2123). Ambos destinados a la carretera. El resto son puentes de ferrocarril: Ios de Lora del Río, Tocina, Mengibar, Alcolea, El Alcalde o Baeza. Todos ellos con amplio desarrollo longitudinal debido a los condicionantes topográficos del terreno.

Por su parte, siguieron construyendose puentes de fábrica. También experimentaron una evolución respecto las obras precedentes. Se rebajaran las bóvedas y en general no se dieron licencias decorativas como en siglos precedentes. Las rasantes serán planas igualadas al nivel del camino. Los tajamares disminuyen de tamaño y serán igual en ambos frentes. Los estribos se reforzaran ante la importancia de su misión sustentadora. Todo siguiendo las enseñanzas heredadas de Perronet. En nuestro río solo tenemos dos ejemplos: el puente del Hacha en la sierra de Cazorla, y un tramo del viejo puente de Andujar hundido durante la Guerra de la Independencia (RECONSTRUCCIÓN: cajas $2340,5743$ y 5744$)$.

\section{LA REVOLUCIÓN CONSTRUCTIVA: ACERO Y HORMIGÓN}

En el siglo XX la construcción de puentes llega a un desarroIlo inusitado. No hubiera sido posible sin la utilización de los nuevos materiales: acero y hormigón. El primero, liberó a las construcciones metálicas de la fragilidad ante la corrosión. El segundo en solitario, en principio, y después armado con metal en su interior, aunará las cualidades de la piedra y el metal. Perteneciente al primer tipo en el Guadalquivir se puede destacar los puentes de Villa del Río, Lora del Río, o el móvil de San Juan de Aznalfarache, todos de carretera, en cuanto al segundo tipo, de hormigón en masa, destacan el puente de Megíbar de la antigua carretera de Bailén a Jaén y el de San Rafael en Córdoba (RUBIATO LACAMBRA, 1997: 523), y de hormigón armado los de Villanueva de la Reina, el de la Cerrada de Utrero en Cazorla, Jaén, y el de San Telmo en Sevilla, entre otros muchos.
El invento del pretensado por el ingeniero francés Eugéne Freyssinet supondrá aún un mayor avance en las posibilidades constructivas del hormigón. Como consecuencia se fabrican las piezas en las márgenes y se montan por medio de voladizos sucesivos. Este sistema fue utilizado por primera vez en 1962 en España sobre el Guadalquivir en el Puente de Almodóvar del Río, obra del ingeniero Carlos Fernández Casado. Se produce así una autentica revolución constructiva que ha permitido la construcción de gran número de carreteras y de puentes. Además de estos novedades, se dio otra línea de actuación, si cabe más sorprendente, las obras mixtas. En las que hormigón y metal colaboran unidos pero no mezclados. Una de las primeras obras de este tipo será la del puente de Posadas sobre el Guadalquivir. Iniciado antes de la Guerra Civil, tras ella, se hizo cargo de su construcción Eduardo Torroja imprimiéndole su audacia y originalidad (PÁEZ BALACA, 1950: 206). Esta técnica ira evolucionando y será aplicada a los nuevos métodos constructivos de viga-cajón y unión de dovelas, consiguiendo bellísimos puentes como el de El Arenal en Córdoba, o el de la autovía en Mengibar.

No se puede acabar esta reflexión del Guadalquivir y sus puentes sin al menos citar la Expo del 92 por la trascendencia que tuvo en dicha cuestión. Es verdad que se centró, afectó y transformó especialmente a Sevilla. Para ello fueron necesarios años de debate hasta que en 1987 se aprobó El Plan General de Obras Urbanas de Sevilla. Con él, además de la apropiación de la isla de La Cartuja como zona urbana, una vez solucionados los problemas de las periódicas inundaciones con las que el río sometía a la ciudad, se construyeron diez puentes. A parte de estos, se realizaron y proyectaron otros en la ciudad de Córdoba, también sobre el Guadalquivir, como el ya citado de El Arenal, el de Miradores, o el de Andalucía y los correspondientes a la transformación de la carretera N-IV en autovía. Pero la explosión constructora de Sevilla no solo fue importante por la cantidad de puentes construidos, sino por la calidad del diseño, la variedad tipológica: Iosas ortótropas, atirantados, arcos metálicos, etc., los magníficos materiales utilizados y los procesos constructivos. Puentes como el de La Barqueta, La Cartuja, Chapina, Las Delicias, El Alamillo, o el Quinto Centenario, por citar solo unos pocos, supusieron un salto cualitativo que ha situado a la ingenieria española en los primeros a nivel mundial.

Hoy, ya en el siglo XXI, el río sigue su fluir. También sigue nuestra actividad, con ella se necesitan nuevas comunicaciones. Estas precisan de nuevos puentes, como el recién construido de la variante de Montoro o el nuevo de Palma del Río. Obras de hoy, que reflejan unas técnicas, un tiempo y una forma de vivir. Sin duda son también una aportación más al ya rico patrimonio histórico de puentes del Guadalquivir.

\footnotetext{
Notas:

${ }^{1}$ Los otros puentes construidos en este periodo en Andalucia fuera del Guadalquivir son los de Benameji, Puente Genil y Ariza.

${ }^{2}$ Mapa de la batalla de Alcolea. Archivo Cartográfico del Ejército, documento 274.
} 